\title{
ENTEROPARASITOSES E SITUAÇÃO NUTRICIONAL DE GESTANTES ATENDIDAS EM UNIDADES BÁSICAS DE SAÚDE DO MUNICÍPIO DE PONTA GROSSA - PR
}

\author{
INTESTINAL PARASITOSIS AND NUTRITIONAL SITUATION OF \\ PREGNANT WOMEN ATTENDED IN BASIC HEALTH UNITS IN PONTA \\ GROSSA - PR
}

\author{
Sandineli BINI ${ }^{2}$, Luciana ALVES', Mackelly SIMIONATTO2 ${ }^{2}$, Júlio César MINÉ \\ ${ }^{1}$ Autor para contato: Departamento de Análises Clínicas e Toxicológicas, Laboratório de \\ Parasitologia Clínica. Universidade Estadual de Ponta Grossa, UEPG. Avenida Carlos \\ Cavalcanti, 4748, Uvaranas, 84030-900, Ponta Grossa, PR, Brasil. E-mail: juliomine@ \\ gmail.com \\ ${ }^{2}$ Departamento de Análises Clínicas e Toxicológicas. Universidade Estadual de Ponta \\ Grossa, UEPG. Avenida Carlos Cavalcanti, 4748, Uvaranas, 84030-900, Ponta Grossa, PR, \\ Brasil.
}

\begin{abstract}
RESUMO
A gestação provoca mudanças orgânicas e psicológicas próprias do processo fisiológico que caracteriza este período. Doenças parasitárias e anemia, quando ocorrem no curso da gestação, devido às características metabólicas, endocrinológicas e imunológicas próprias, podem determinar preocupações e por isso o exame coproparasitológico deve ser recomendado. $\mathrm{O}$ objetivo do presente trabalho foi determinar a frequência de enteroparasitos e a situação nutricional em gestantes de Ponta Grossa - PR, por meio de análises antropométricas e laboratoriais. Tais dados foram relacionados com respostas obtidas dos questionários estruturados a respeito de características sócio-econômicoculturais e sanitárias das gestantes. Foram convidadas 101 gestantes atendidas em Unidades Básicas de Saúde, das quais 21 participaram com amostras clínicas. Das 21 gestantes, $23,80 \%$ estavam parasitadas, a maioria encontrava-se em peso normal na gestação $(42,86 \%)$ e $33,33 \%$ estavam com anemia sem que estivessem parasitadas concomitantemente. Pode-se observar que a relação entre situação nutricional e enteroparasitoses em gestantes não é um problema no município, mas os resultados dos índices antropométricos, hemograma e dos exames coproparasitológicos, analisados isoladamente, mostram necessidade de vigilância.
\end{abstract}

Palavras-chave:Enteroparasitos, Situação Nutricional, Gestantes, Análises laboratoriais.

\begin{abstract}
Pregnancy causes organic and physiological changes specific to the psychological process that characterizes this period. Parasitic diseases and anemia that happen in the course of pregnancy raise concerns due to metabolic, endocrinological and immunological characteristics, and, because of this, the stool examinations must be done. The goal of this study was to determine the frequency of intestinal parasites and nutritional status of pregnant women in Ponta Grossa - PR, using anthropometric and laboratory analysis. These data were related to answers obtained from structured questionnaires regarding socio-economic, cultural and health characteristics of pregnant women. One hundred and one pregnant women attended in Basic Health Units were invited to this survey, from which 21 participated with clinical samples. Of this 21 pregnant women, $23.80 \%$ were parasitized, the majority were in normal weight during pregnancy $(42.86 \%)$ and
\end{abstract}


$33.33 \%$ were anemic without being parasitized simultaneously. It can be observed that the relationship between nutritional status and presence of intestinal parasites in pregnant women is not a problem in Ponta Grossa, but the results of anthropometric index, blood tests and fecal examinations, analyzed individually, show that they have to be monitored.

Key-words: Intestinal Parasitosis, Nutritional Status, Pregnancy, Laboratorial Analysis.

\section{INTRODUÇÃO}

Sabe-se que a gestação provoca mudanças orgânicas e psicológicas próprias do processo fisiológico caracterizador deste período. As mulheres em período gestacional representam um grupo de indivíduos com características próprias e transitórias dos padrões metabólicos, endocrinológicos e imunológicos e requerem cuidados especiais quando se apresentam portadoras de doenças parasitárias (MACEDO; REY, 1996).

O estado nutricional afeta a saúde da gestante, pois ela, o recém-nascido e inclusive o aleitamento materno estão diretamente relacionados com o estado nutricional antes ou durante a gestação. Para essa avaliação, podem-se utilizar dados de anamnese (história pessoal, social, clínica e obstétrica), antropometria - medida do tamanho corporal e suas proporções, indicador direto do estado nutricional - testes laboratoriais e dados sobre a ingestão alimentar da gestante. Os testes bioquímicos também são empregados, pois oferecem determinações objetivas e precisas a respeito das concentrações de nutrientes dos tecidos, sangue ou urina, apesar de na gestação ocorrerem alguns problemas de interpretação devido às alterações fisiológicas e ausência de normas estabelecidas para cada período específico dela. Porém, os achados laboratoriais oferecem uma linha básica para o monitoramento do estado nutricional (PONTES, 2007).

A anemia é definida pela Organização Mundial da Saúde (OMS) como a condição na qual o conteúdo da hemoglobina do sangue está abaixo dos valores considerados normais para a idade, sexo, o estado fisiológico e a altitude, sem considerar a causa da deficiência. (ALMEIDA, 2007). Para Cantos, Dutrae Koerick (2003), entre os fatores determinantes do tipo de anemia estão certos parasitas intestinais, que podem reduzir em até $20,00 \%$ o ferro ingerido na dieta, sendo que a causa orgânica imediata é a deficiência de ferro circulante.
Clinicamente, as enteroparasitoses podem cursar com sintomas digestivos leves, como dor abdominal, náuseas, diarreia ou constipação intestinal, digestão difícil e flatulência, podendo, em algumas ocasiões, esses sintomas, serem confundidos com manifestações próprias do início da gravidez. Em determinados casos, as enteroparasitoses podem ser a causa de uma anemiaque não responde ao tratamento clínico rotineiro, a anemia materna por deficiência de ferro tem repercussões sobre o concepto levando a restrição do crescimento intrauterino, prematuridade, baixo peso ao nascer, baixas reservas fetais de ferro e mortalidade materna, fetal e neonatal aumentada(SOUZA et al., 2002).

Os parasitos mais associados à anemia ferropriva são os ancilostomídeos (Ancylostomaduodenale e Necatoramericanus), vermes que espoliam de $0,05 \mathrm{~mL}$ a $0,3 \mathrm{~mL}$ e $0,01 \mathrm{~mL}$ a $0,04 \mathrm{mLde}$ sangue/ dia, respectivamente; Trichuristrichiura, que pode danificar a mucosa intestinal, causando erosões e ulcerações múltiplas, ingerindo cerca de $0,005 \mathrm{~mL}$ de sangue/dia; o Strongyloidesstercoralis, que provoca pontos hemorrágicos e ulcerações de vários tamanhos na submucosa do intestino delgado, devido a sua penetração na mesma; a amebíase, provocada pela Entamoebahistolytica, em sua forma invasiva, mais frequentemente causa disenteria aguda, perfuração do intestino grosso e hemorragias; o Ascaris lumbricoides e a Giardialamblia exercem caráter secundário em associação à anemia, causando, ambos, diminuição da absorção de ferro e vitaminas(NEVES, 2005).

São estimadas 1,6 bilhões de pessoas anêmicas ao redor do mundo e essa situação é comum durante a gestação. Aproximadamente $50,00 \%$ das gestantes têm anemia. A deficiência de ferro é a principal causa de anemia e suas consequências na gestação tem sido bem estabelecidas, incluindo mortalidade materna e fetal e baixo peso ao nascer. Em gestantes a anemia mais comumente classificada é a ferropriva, uma condição que pode ser definida como a redução patológica da 
taxa de hemoglobina circulante a um valor inferior a 12 g/dLpara mulheres (ROCHA et al., 2004).Evidências suportam a relação entre deficiência de ferro e "déficit" do desenvolvimento cognitivo em crianças e capacidade de trabalho reduzida em adultos. A suplementação de ferro e ácido fólico é uma assistência pré-natal padrão em muitos países, baseada em seus benefícios na prevenção da anemia materna e complicações relacionadas (FINKEISTEIN et al., 2012).

Reconhecendo os efeitos prejudiciais da anemia para a saúde e sobrevida do binômio mãe/filho, o Ministério da Saúde implantou a suplementação medicamentosa de ferro no Brasil pelo Programa de atenção à Gestante em 1982 e a reiterou, em 2005, com o Programa Nacional de Suplementação de Ferro por meio da Portaria n. ${ }^{\circ} 730$, de 13 de maio de 2005 (BRASIL, Ministério da Saúde. Portaria..., 2005).

A gestação também está incluída na lista dos fatores clássicos desencadeantes da obesidade. O início ou manutenção da obesidade nesta fase está associado a inúmeros riscos maternos e fetais. Numa gestação normal o ganho de peso ocorre devido ao aumento de tecidos maternos e dos produtos da concepção. O ganho de peso ideal na gestação é baseado nas recomendações do Instituto de Medicina e Conselho Nacional de Pesquisa - Washington - USA (IOM \& NRC, 2009) e leva em consideração o IMC pré-gravídico da paciente. No Brasil, o Ministério da Saúde adota recomendações sobre o ideal para o ganho total de peso, no estado nutricional inicial da gestante, classificando de acordo com categorias de índice de massa corporal (IMC) por semana gestacional (BRASIL, Ministério da Saúde. Pré-natal...,2005).

A hiperglicemia da gestante resulta em hiperglicemia do feto, com consequente aumento na produção de insulina, resultando em hipertrofia e hiperplasia das células $\beta$-pancreáticas. Esse estado resulta em hiperinsulinismo e excesso de glicose intra-uterino, comumente encontrado em gestações que se complicam pelo diabetes, favorecendo a macrossomia fetal (LANGER, 2000). A macrossomia é acaracterística mais comumente encontrada entre mulheres multíparas, com no mínimo 30 anos de idade, maior estatura e índice elevado de massa corporal (IMC), além disso, histórico familiar de diabetes e de macrossomia fetal colaborampara esta condição (RUDGE; CALDERON, 1997).

Segundo Nogueiraet al. (2011), as gestantes classificadas com diabetes gestacional (DMG) devem apresentar glicemia de jejum, na sua primeira consulta pré-natal, com valores $\geq 92 \mathrm{mg} / \mathrm{dL}$, mas $<126 \mathrm{mg} / \mathrm{dL}$. Além disso, para demonstrar o estado prejudicial que a gestante classificada com DMG pode desenvolver, aproximadamente $7,00 \%$ das gestações são complicadas pelo estado de DMG. A identificação de mulheres que desenvolveram diabetes na gestação possibilita não apenas minimizar os efeitos adversos que essa desordem metabólica traz para mãe e filho, mas colabora para identificar mulheres com risco aumentado para desenvolver diabetes no futuro.

Dessa forma, com o desenvolvimento desse trabalho,pôde-se determinar, por meio das metodologias descritas a seguir, a frequência de enteroparasitos em um grupo de gestantes do município de Ponta Grossa - PR, bem como se estabeleceu a relação entre os dados obtidos pela análise de questionário estruturado para obtenção de informações relacionadas às características sócio-econômico-culturais e sanitárias das gestantes com os resultados dos exames hemato-bioquímicos, fornecendo dados para melhor entender as questões relacionadas com a situação nutricional de gestantes desse município.

\section{METODOLOGIA}

Em 2012, Ponta Grossa atendia em média cinco mil gestantes(BUSNARO, 2012). Durante o período gestacional, são feitas, no mínimo, sete consultas pré-natais, com exames de rotina e ultra-sonografia. As mães atendidas pela ESF (Estratégia Saúde da Família) do município tiveram a oportunidade de participar do grupo de gestantes, em que receberam orientações sobre saúde, alimentação e exercícios físicos. As equipes procuram dar o atendimento a todas as gestantes em que a ESF atua e quando problemas na gravidez eram detectados, a gestante foi encaminhada para o Centro Municipal da Mulher (CMM), onde se faz o acompanhamento médico por especialistas com intervalos menores do que os realizados nas Unidades Básicas de Saúde (UBS) (BUSNARO, 2012).

O presente estudo obteve autorização da Secretaria Municipal de Saúde de Ponta Grossa, após reunião para explanação sobre o mesmo com o secretário municipal de saúde da época e obteve aval do Comitê de Ética em Pesquisa da UEPG em 19/12/2013 (protocolo $\mathrm{n}^{\mathrm{o}}$ 501.171).

No período compreendido entre janeiro e julho de 2014 foi estudada uma amostra por conveniência de 
101 gestantes provenientes de quatro UBSs para responder a um questionário estruturado afim de averiguar dados relacionados às condições sócio-econômico-culturais e de saneamento em que tais gestantes viviam, além disso, foram medidos os dados antropométricos, e as gestantes forneceram amostras biológicas de sangue e de fezes para realização das análises laboratoriais.

A pesquisa em questão foi apresentada às gestantes quando as mesmas chegavam à UBS para consulta com o médico e, então, um convite era feito para que as mesmas participassem do projeto. Quando as gestantes aceitavam participar, assinavam um Termo de Consentimento Livre e Esclarecido (TCLE), ficavam cientes de que suas identidades seriam preservadas, respondiam um breve questionário e recebiam um frasco coletor de fezes, um lembrete com data, local e hora para que fosse trazida a amostra de fezes e realizada a coleta do sangue periférico com jejum prévio.

As gestantes informaram no questionário seu peso anterior à gestação para a avaliação antropométrica, além da sua altura. Em alguns casos, quando as gestantes não recordavam seus dados, examinavam-se suas Carteirinhas da Gestante, oferecida pela Unidade de Saúde, com a devida permissão. O Índice de Massa Corporal pré-gravídico foi então calculadopor meio da fórmula: IMC $=$ Peso $(\mathrm{kg}) /\left(\right.$ Estatura $^{2}(\mathrm{~m})$, segundo Rezende et al. (2010) e classificação de acordo com a Diretriz de Sobrepeso e Obesidade da Sociedade Brasileira de Endocrinologia e Metabologia (SBEM, 2004).

Para todas as gestantes participantes conferiram-se os dados de peso atual e estatura, utilizando balança antropométrica de plataforma. Com base nos dados obtidos, utilizou-se a curva de peso/idade gestacional e nomograma segundo Rosso e Mardones (1986) para obter-se a categoria do estado nutricional de cada gestante.

As amostras fecais recebidas assim como o sangue coletado foram imediatamente enviados aos Laboratórios de Parasitologia, Hematologia e Bioquímica do Laboratório Universitário de Análises Clínicas (LUAC) da Universidade Estadual de Ponta Grossa (UEPG).Asfezes foram processadas e analisadas conforme metodologia de sedimentação simples segundo Hoffmann, Pons e Janer (1934); centrifugo-flutuação em sulfato de zinco segundoFaustet al. (1939); técnica pautada no termohidrotropismo de larvas de nematódeos segundo Rugai, Mattos e Brizola (1954) e técnica de coloração de Kinyoun segundo Martinez e Belda Neto (2001) após centrífugo-sedimentação para pesquisa de parasitos coccídeos intestinais.

O hemograma completo foi realizado em amostra de sangue total obtida por punção venosa após autorização da paciente ou responsável (para os casos de gestantes menores de 18 anos). A avaliação laboratorial do eritrograma e do leucograma realizou-se, respectivamente, de acordo com a metodologia semi-automatizada por contador eletrônico de múltiplos canais de Hematologia e Contagem Diferencial de Leucócitos manual por microscopia de distensão corada (FAILACE, 1995).

A determinação da glicemia de jejum das gestantes foi realizada por meio do kit "Glicose GOD-PAP Kovalent ${ }^{\circledR}$ " em amostra de sangue coletada em tubo com fluoreto de sódio (GLICOSE GOD-PAP, 2010).

Para a determinação de ferro sérico, utilizou-se o kit "Ferro FereneKovalent $\AA$ " em amostra de soro em tubo com gel separador. (FERRO FERENE, 2008).

Simultaneamente à aplicação do questionário, foram realizadas atividades educativas de forma individual e coletiva, com informações verbais acerca das doenças parasitárias e profilaxia das mesmas, enfatizando a importância das análises laboratoriaisno período gestacional, além da entrega de um folder contendo informações básicas sobre parasitoses.

Os laudos dos exames laboratoriais foram emitidos e entregues às gestantes participantes na respectiva Unidade Básica de Saúde.

Os resultados provenientes desse estudo foram analisados por meio de estatística descritiva e expressos em frequências absolutas e relativas.

\section{RESULTADOS}

Das 101 gestantes convidadas, 21 realizaram a coleta de sangue e trouxeram a amostra de fezes para prosseguimento das análises laboratoriais. Foram selecionados dois grupos com as gestantes participantes do estudo: o primeiro, formado pelas que responderam aos questionários e fizeram as medidas antropométricas (101 gestantes)(G1) e o segundo grupo, formado pelas gestantes que além de terem respondido aos questionários e realizado as medidas antropométricas, trouxeram as amostras biológicas para realização dos exames laboratoriais (21 gestantes) (G2).

Alguns dados coletados pela análise dos questionários sobre condições sócio-econômico-culturais 
das gestantes encontram-se na Tabela 1 em que se pode depreender que a maioria das gestantes apresentou idade de 20 a 24 anos $(32,67 \%)$, os períodos gestacionais mais frequentes foram o segundo e terceiro trimestres (72,27\%), o nível de escolaridade mais apresentado pelas gestantes foi Ensino Médio completo e Ensino Médio incompleto $(51,48 \%)$, a maioria das gestantes mostrou possuir renda familiar de 1 a 2 salários mínimos $(85,15 \%)$ e moradia em zona urbana $(96,04 \%)$. Entre as gestantes que participaram integralmente do estudo $(n=21)$, disponibilizando suas amostras biológicas, a maioria tinha idade entre 20 e 29 anos (57,14\%). Quanto ao período gestacional, a maioria das gestantes desse grupo encontrava-se no segundo trimestre de gestação $(66,67 \%)$. Em contraste com o primeiro grupo, este apresentou gestantes com menor escolaridade. Assim como no primeiro grupo de gestantes, a renda familiar mais comum foi na faixa de 1 a 2 salários mínimos $(76,19 \%)$ e estas possuem residências na zona urbana $(95,24 \%)$.

Com relação a outros dados obtidos pela análise dos questionários, a maioria das gestantes do primei- ro grupo informou residir em casa própria $(59,41 \%)$, assim como as gestantes do segundo grupo $(66,67 \%)$.

No que diz respeito ao saneamento e hábitos higiênicos a maioria das gestantes, dos dois grupos afirmou que existe saneamento básico, esgoto tratado e água tratada em suas residências $(89,11 \%$ e $80,95 \%$, respectivamente), porém, há gestantes que referem ausência de saneamento básico nos locais onde moram (2,97\% no primeiro grupo de gestantes e $14,29 \%$ no segundo grupo de gestantes). Com relação aos hábitos higiênicos, no primeiro grupo, 96,04\% afirmaram lavar as mãos antes das refeições e após irem ao banheiro, $1,98 \%$ após ir ao banheiro e $0,99 \%$ antes das refeições. Uma pequena parcela desse mesmo grupo $(0,99 \%)$ afirmou que "às vezes" lavava as mãos antes das refeições e após ir ao banheiro, porém, 47,52\% disseram andar descalças. No segundo grupo, 95,24\% afirmaram lavar as mãos antes das refeições e após ir ao banheiro e apenas uma gestante afirmou lavar as mãos após ir ao banheiro. Deste grupo, 42,86\% afirmaram andar descalças.

Tabela 1 - Dados obtidos pelas respostas do questionário estruturado aplicado às gestantes - Ponta Grossa - 2014

\begin{tabular}{|c|c|c|c|c|}
\hline \multirow{2}{*}{ Variáveis } & \multicolumn{2}{|c|}{ Grupo 1 (Todas as gestantes) } & \multicolumn{2}{|r|}{ Grupo 2 (Gestantes que fizeram exames laboratoriais) } \\
\hline & $N$ & $\%$ & $n$ & $\%$ \\
\hline \multicolumn{5}{|l|}{ Idade } \\
\hline$<20$ & 23 & 22,77 & 4 & 19,05 \\
\hline $20-24$ & 33 & 32,67 & 6 & 28,57 \\
\hline $25-29$ & 29 & 28,71 & 6 & 28,57 \\
\hline $30+$ & 16 & 15,84 & 5 & 23,81 \\
\hline \multicolumn{5}{|l|}{ Períodogestacional } \\
\hline $1^{\circ}$ trimestre & 28 & 27,72 & 5 & 23,81 \\
\hline $2^{\circ}$ trimestre & 38 & 37,62 & 14 & 66,67 \\
\hline $3^{\circ}$ trimestre & 35 & 34,65 & 2 & 9,52 \\
\hline \multicolumn{5}{|l|}{ Escolaridade } \\
\hline Ensino Fundamental incompleto & 19 & 18,81 & 6 & 28,57 \\
\hline Ensino Fundamental completo & 20 & 19,8 & 5 & 23,81 \\
\hline EnsinoMédioincompleto & 26 & 25,74 & 5 & 23,81 \\
\hline EnsinoMédiocompleto & 26 & 25,74 & 2 & 9,52 \\
\hline Ensino Superior completo & 6 & 5,94 & 0 & 0 \\
\hline \multicolumn{5}{|l|}{ Renda familiar } \\
\hline$<1$ saláriomínimo & 4 & 3,96 & 1 & 4,76 \\
\hline 1 a 2 saláriosmínimos & 86 & 85,15 & 16 & 76,19 \\
\hline 3 a 5 saláriosmínimos & 9 & 8,91 & 3 & 14,29 \\
\hline$>5$ saláriosmínimos & 2 & 1,98 & 1 & 4,76 \\
\hline \multicolumn{5}{|l|}{ Região } \\
\hline Zona urbana & 97 & 96,04 & 20 & 95,24 \\
\hline Zona rural & 4 & 3,96 & 1 & 4,76 \\
\hline
\end{tabular}

Fonte: Os autores. 
Quanto aos dados antropométricos das gestantes obtidos nos questionários, de acordo com a metodologia citada, verificou-se que, tanto no primeiro grupo de gestantes quanto no segundo grupo, a maioria apresentou peso normal (eutróficas) em relação ao peso pré-gravídico. Quanto à curva de peso e idade gestacional, em que se considerou o peso da gestante referente ao período gestacional do momento da entrevista, observou-se a mesma frequência de gestantes com peso normal e com obesidade no primeiro grupo $(31,68 \%)$. No segundo grupo de gestantes avaliado, a maioria encontrava-se com peso normal na gestação $(42,86 \%)$. Estes e os demais resultados podem ser vistos na Tabela 2.

Tabela 2 - Dados antropométricos relacionados às gestantes - Ponta Grossa/2014

\begin{tabular}{l|l|l|l|l}
\hline \multirow{2}{*}{ Variáveis } & \multicolumn{2}{l|}{$\begin{array}{l}\text { Grupo 1 } \\
\text { (Gestantes com amostra) }\end{array}$} & \multicolumn{2}{l}{$\begin{array}{l}\text { Grupo 2 } \\
\text { (Gestantes que fizeram exames laboratoriais) }\end{array}$} \\
\cline { 2 - 5 } & $n$ & $\%$ & $N$ & $\%$ \\
\hline
\end{tabular}

\section{Classificação IMC pré-gravídico}

\begin{tabular}{l|c|l|c|l}
\hline Baixo peso & 8 & 7,92 & 1 & 4,78 \\
\hline Peso normal & 49 & 48,51 & 11 & 52,38 \\
\hline Pré-obesidade & 26 & 25,74 & 7 & 33,33 \\
\hline Obesidade I & 15 & 14,85 & 2 & 9,52 \\
\hline Obesidade II & 2 & 1,98 & 0 & 0,00 \\
\hline Obesidade III & 1 & 0,99 & 0 & 0,00 \\
\hline
\end{tabular}

\section{Curva de peso/idade gestacional}

\begin{tabular}{l|l|l|l|l}
\hline Baixo & 23 & 22,77 & 4 & 19,05 \\
\hline Normal & 32 & 31,68 & 9 & 42,86 \\
\hline Sobrepeso & 14 & 13,86 & 2 & 9,52 \\
\hline Obesidade & 32 & 31,68 & 6 & 28,57 \\
\hline
\end{tabular}

Fonte: Os autores.

Quando indagadas sobre a realização de suplementação com sulfato ferroso e ácido fólico durante a gestação, a maioria, em ambos os grupos afirmou que havia feito uso de tais suplementos em algum período gestacional $(72,28 \%$ no primeiro grupo e $76,19 \%$ no segundo grupo). Nos dois grupos de gestantes avaliados, quando se perguntou sobre casos de diabetes na família, a maioria relatou histórico familiar para essa doença $(60,40 \%$ no primeiro e $71,43 \%$ no segundo grupo de gestantes).

Tabela 3 - Relação entre os resultados do Hemograma e Exame Parasitológico de Fezes (EPF) de gestantes -Ponta Grossa - 2014

\begin{tabular}{l|l}
\hline Exames Laboratoriais & $\boldsymbol{n ( \% )}$ \\
\hline Hemograma normal e EPF negativo & $9(42,87)$ \\
\hline Hemograma normal e EPF positivo & $4(19,04)$ \\
\hline Hemograma alterado e EPF negativo & $7(33,33)$ \\
\hline Hemograma alterado e EPF positivo & $1(4,76)$ \\
\hline Total & $21(100,00)$ \\
\hline
\end{tabular}

Fonte: Os autores.
Após a realização dos exames laboratoriais foram emitidos os laudos e os resultados foram analisados. Os resultados da correlação entre o hemograma completo e exame parasitológico de fezes (EPF) podem ser vistos na Tabela 3 e os parasitos encontrados estão descritos na Tabela 4.

Considerou-se como hemograma alterado, a análise em que houve um ou mais valores no eritrograma (hemácias, hemoglobina, hematócrito, volume corpuscular médio (VCM), hemoglobina corpuscular média (HCM) e/ou concentração de hemoglobina corpuscular média (CHCM) abaixo dos intervalos de referência contidos no laudo. Do total de gestantes voluntárias,oito apresentaram alteração no hemograma (valor de hemoglobina abaixo do valor de referência, ou seja, encontravam-se anêmicas). Além da anemia, quatro gestantes apresentaram hematócrito alterado, duas CHCM abaixo do valor de referência, uma hematócrito e CHCM abaixo do valor de referência e uma com VCM e CHCM abaixo dos valores de referência. O leucograma também foi analisado: quatro gestan- 
tes $(19,05 \%)$ apresentaram valor acima do intervalo de referência para leucócitos, nenhuma apresentava eosinofilia e a presença de granulações tóxicas nos neutrófilos foi observada em três gestantes $(9,52 \%)$.

De acordo com os dados da Tabela 3 pode-se notar, então, que a maioria dos casos (13) apresentou hemograma normal e oito apresentaram hemograma alterado. A maioria das voluntárias apresentou EPF negativo (16 gestantes) e cinco gestantes apresentaram positividade para esta análise.

Com relação aos exames bioquímicos, dois parâmetros foram avaliados: glicemia de jejum e determinação de ferro sérico, sendo que todas as gestantes apresentaram exames com valores referenciais normais para estas análises.

Tabela 4 - Parasitosencontrados nos Exames Parasitológicos de Fezes realizados - Ponta Grossa - 2014

\begin{tabular}{l|l|l}
\hline Parasitos & $\boldsymbol{n}$ & $\mathbf{\%}$ \\
\hline Enterobiusvermicularis & 1 & 4,76 \\
\hline Hymenolepis nana & 1 & 4,76 \\
\hline Endolimax nana & 1 & 4,76 \\
\hline Entamoeba coli & 1 & 4,76 \\
\hline Entamoeba coli eEndolimax nana & 1 & 4,76 \\
\hline Negativos & 16 & 76,19 \\
\hline Total & 21 & 100,00 \\
\hline
\end{tabular}

Fonte: Os autores.

Quanto aos resultados encontrados na Tabela 4 , obtivemos ao todo $23,81 \%$ dos resultados positivos para alguma espécie de enteroparasito, incluindo o grupo dos helmintos e dos protozoários. Dentre os resultados positivos, ocorreu um caso de biparasitismo com espécies de protozoários não patogênicos (Entamoeba coli e Endolimax nana).A maioria das gestantes $(76,19 \%)$ não albergava nenhum parasito.

Nenhum oocisto dos protozoários emergentes Cryptosporidiumsp., Cyclosporacayetanensis e Cystoisospora belli foram detectados por meio da coloração de Kinyoun.

\section{DISCUSSÃO}

Um estudo conduzido na Tanzania por Finkeisteinet al. (2012) relacionou infecções parasitárias, anemia e deficiência de ferro em gestantes HIV positivas. Verificou-se que $82,50 \%$ das gestantes eram anêmicas (considerando-se $11 \mathrm{~g} / \mathrm{dL}$ como valor de referência para hemoglobina) e $26,40 \%$ encontrava- -se em quadro de anemia severa, com hipocromia e microcitose leve e moderada, respectivamente. Nesse mesmo estudo, a presença de protozoários patogênicos foi associada com significativo aumento no risco de desenvolvimento de anemia durante o período de acompanhamento (904 mulheres tiveram valor de hemoglobina analisado durante a gestação e ao menos uma medida após esse período).Quanto às parasitoses, neste estudo, os autores encontraram $24,40 \%$ de positividade para infecções parasitárias (protozoários patogênicos ou helmintos), sendo que as espécies observadas foram: Giardialamblia, Entamoebahistolytica, Cryptosporidiumsp., Trichuristrichiura, Ascaris lumbricoides, Strongyloidesstercoralis, Schistosoma mansonie ancilostomídeos. Em contraste, nopresente estudo foram encontradas espécies de protozoários e helmintos não relatadas por Finkesistein e colaboradores: Entamoeba coli, Endolimax nana, Enterobiusvermicularis e Hymenolepis nana, que mais denotam a ausência de boas condutas higiênicas pessoais e dos alimentos/água consumidos do que a possibilidade desses enteroparasitos causarem anemia.

Em Araraquara, SP, foi realizado um estudo que mostrou que Entamoeba coli é o protozoário não patogênico mais encontrado nas fezes de gestantes (MINÉ, 2009). Tal resultado é coincidente com o aqui apresentado, em que aproximadamente $10,00 \%$ das gestantes que participaram do estudo apresentaram tal parasito nas fezes.

Egwunyengaet al.(2001), mostram que dado o alto índice de fertilidade, estado nutricional deficiente e más condições de higiene nos países em desenvolvimento, as helmintíases podem contribuir significantemente para a situação de anemia em gestantes, além de que essa ocorrência entre elas é um indicativo de poluição fecal do solo e da água de uso doméstico, que são relacionados com falta de saneamento e disposição imprópria de esgoto. Neste estudo conduzido na Nigéria, a prevalência de infecção com helmintos intestinais entre as gestantes avaliadas foi de 48,30\%. A frequência dos helmintos encontrados nas fezes foram:ancilostomídeos (14,30\%), Ascaris lumbricoides $(19,10 \%)$, Trichuristrichiura $(7,00 \%)$, Schistosoma mansoni $(3,40 \%)$, Enterobiusvermicularis $(2,00 \%)$, Hymenolepis $\mathrm{sp}$. $(1,60 \%)$ e Taeniasp. (1,00\%).Dessa forma, pode-se depreender que, em Ponta Grossa, a infecção de gestantes pelos helmintos Enterobiusvermicularise 
Hymenolepisnanaocorre em frequência um pouco superior à encontrada na Nigéria ( $4,76 \%$ cada uma delas).

As análises laboratoriais das gestantes demonstraram que a causa de anemia não está relacionada com a presença de enteroparasitos, visto que as gestantes com EPF positivo(23,80\%) não obtiveram dados hematológicos sugestivos para um estado anêmico, exceto um caso onde houve biparasitismo com as espécies Entamoeba coli e Endolimaxnana. Porém,a anemia desta gestante não se justifica pela presença dos parasitos, já que estas não são consideradas espécies patogênicas.

Segundo Yatichet al.(2009), os fatoresidade menor que 20 anos, baixa renda e a inexistência de relacionamento formal, foram independentemente associados com o aumento da probabilidade de infecção parasitárias em gestantes de Gana.Nesse mesmo estudo, a prevalência de $25,70 \%$ foi observada para infecções parasitárias intestinais, o que pode indicar condições de saneamento precárias, já que $37,90 \%$ das participantes não tinham instalações sanitárias em suas residências.

Em Ponta Grossa, verificou-se que a maioria das gestantes participantes do segundo grupo reside em locais com rede de esgoto e tratamento de água adequado $(80,95 \%)$, dado este, que leva a pensar que o problema relacionado às parasitoses encontradas nas gestantes estão mais atrelados com hábitos duvidosos de higiene pessoal e higienização da água e dos alimentos. As gestantes com hemograma alterado encontradas no presente estudo, parasitadas ou não, possuíam saneamento básico e afirmaram ter condições de higiene adequados.

Especificamente em relação às cinco gestantes parasitadas encontradas nesse estudo $(23,80 \%)$, dado semelhante ao encontrado por Yatichet al. (2009), todas com renda familiar de um a dois salários mínimos e que têm como ocupação atividades domésticas, nenhuma possuía precariedade em relação a questões de saneamento básico e hábitos de higiene, apesar de que três das cinco gestantes parasitadas afirmaram que costumam andar descalças. Vale a pena observar neste ponto que os dados relacionados aos hábitos de higiene podem não ser suficientemente confiáveis, pois ao serem indagadas a respeito desse fato, as pessoas podem sentir-se desconfortáveis ao explanar a realidade.

As gestantes deste estudo que fazem parte do segundo grupo $(n=21)$ não obtiveram dados laboratoriais que acusassem diabetes gestacional apesar de, em sua grande maioria, apresentarem histórico familiar para diabetes.

No estudo de Lima e Sampaio (2004), a classificação do estado nutricional pré-gestacional segundo o IMC mostra que mais da metade das gestantes estavameutróficas $(64,80 \%)$, enquanto que as com baixo peso, sobrepeso e obesidade representaram, respectivamente, $14,80 \%, 16,70 \%$ e $3,70 \%$. Nas gestantes de Ponta Grossa, voluntárias nesse estudo, em relação ao IMC pré-gravídico, a maioria das gestantes do primeiro grupo também se encontravam eutróficas $(48,51 \%)$, semelhantemente às gestantes do segundo grupo $(52,38 \%)$. Como se pode notar na Tabela 2 , a quantidade de gestantes com baixo peso pré-gestacional em cada grupo mostra-se inferior à do estudo citado e os valores referentes ao sobrepeso e à obesidade (graus I, II e III) somados, não superam o grupo das gestantes eutróficas, porém, para efeito de prevenção se faz necessária a constante vigilância dessas gestantes.

Um estudo realizado por Rocha et al.(2005) mostrou que gestantes obesas com baixo nível socioeconômico, possuíam problemas com relação à situação nutricional. Neste mesmo estudo, 43,00\% das gestantes iniciaram o pré-natal com baixo peso, sobrepeso ou obesidade, além disso, das gestantes que iniciaram o pré-natal com baixo peso, $60,00 \%$ apresentavam ganho de peso insatisfatório. No presente trabalho, $22,77 \%$ de gestantes do primeiro grupo apresentavam baixo peso gestacional e no segundo grupo, $19,05 \%$. Os resultados das análises laboratoriais destas gestantes mostram que somente uma encontrava-se anêmica além de apresentar-se com baixo peso e nenhuma albergava parasitos, mostrando que o estado subnutrido provavelmente não é devido às parasitoses.

Na pesquisa realizada por Padilha et al. (2007), apesar da maior parte da amostra estar relacionada a gestantes eutróficas $(53,70 \%)$, houve uma proporção expressiva de gestantes com sobrepeso, seguida de baixo peso e obesidade. Fato inverso ocorreu no estudo de Hedrichet al. (2007) no qual foi observado que a maioria das gestantes apresentava baixo peso. De acordo com os resultados obtidos no presente estudo, tendo como parâmetro a curva de peso/idade gestacional, o primeiro grupo de gestantes apresentou-se em igual frequência para peso normal e obesidade $(31,68 \%)$, seguida das que estavam com baixo peso e sobrepeso, respectivamente (Tabela 2). Já no segundo grupo de gestantes, a grande maioria se encontrava eutrófica 
(42,86\%), seguida das que estavam obesas, com baixo peso e com sobrepeso. Esses resultados se coadunam aos encontrados por Padilha et al. (2007).

Há necessidade de acompanhamento mais eficiente do ganho de peso durante a gestação e atendimento nutricional não apenas para as gestantes com baixo peso, mas para todas, contribuindo assim para evitar excesso ou déficit de peso ao final da gestação e reduzindo ao mínimo os riscos obstétricos e de obesidade pós-parto (LIMA; SAMPAIO, 2004).

Torna-se evidente a importância do aprofundamento de estudos que demonstrem o quão prejudicial pode se tornar a presença de enteroparasitos em grupos especiais (como o das gestantes), envolvendo a situação nutricional e sócio-econômica nesta fase da vida, em que se fazem necessários cuidados redobrados, com o intuito de preservar a saúde de mãe e filho. Além da hemodiluição que corrobora para uma anemia discreta, causada pela demanda de ferro para oxigenar o feto, uma vez que o volume plasmático e massa eritrocitária não são proporcionais, pois o volume plasmático é maior do que a massa eritrocitária até o final da gestação, onde essa desproporção se estabiliza (SOUZA; BATISTA FILHO; FERREIRA,2002).

\section{CONCLUSÃO}

De acordo com os resultados apresentados pode-se observar que a relação entre situação nutricional de gestantes e a presença de parasitoses não se mostrou como um problema no município de Ponta Grossa, porém os índices antropométricos (IMC pré-gestacional e curva peso/idade gestacional), resultados de hemograma e de exames parasitológicos analisados de maneira isolada mostram a necessidade de constante vigilância das gestantes que, mesmo com baixas, porém consideráveis frequências vivem em condições inadequadas de saneamento, possuem parasitoses intestinais, anemia e encontram-se com sobrepeso ou obesidade, situações que colaboram para o desenvolvimento de problemas mais graves de saúde tanto para a mãe quanto para o concepto.

\section{AGRADECIMENTOS}

Às gestantes que colaboraram para a realização deste estudo, à Secretaria Municipal de Saúde de Ponta Grossa, à Profa. Dra. Cláudia Solano Rocha pela análise estatística realizada e à Profa. Stella de Medeiros Ivahy Badaró pela correção do idioma inglês.

\section{REFERÊNCIAS}

ALMEIDA, J. L. V. Prevalência de anemia ferropriva associada a fatores de risco em pré-escolares da creche Cantinho do Fiorello no município de Natividade - RJ. NewsLab, São Paulo, 84 ed., 2007.

BRASIL. Ministério da Saúde. Portaria n. ${ }^{\circ} 730$, de 13 de maio de 2005. Institui o Programa Nacional de Suplementação de Ferro, destinado a prevenir a anemia ferropriva e dá outras providências. Diário Oficial da União. Brasília, 2005.

BRASIL. Ministério da Saúde. Pré-natal e puerpério: atenção qualificada e humanizada - Manual Técnico. Secretaria de Atenção à Saúde, Departamento de Ações Programáticas Estratégicas. Área Técnica de Saúde da Mulher. Brasília, 2005.

BUSNARO, E. Prefeitura reduz mortalidade materna em 76\%. Portal da Prefeitura de Ponta Grossa,Ponta Grossa,set. 2012. Disponível em<http://www.pontagrossa. pr.gov.br/node/13083>. Acesso em: 18 set. 2014.

CANTOS, G. A.; DUTRA, R. L; KOERICK, J. P. Ocorrência de anemia ferropriva em pacientes com enteroparasitoses. Saúde em revista, Piracicaba, v. 5, n. 10, p. 1-79, 2003.

EGWUNYENGA, A. O. et al. Plasmodium/intestinal helminthco-infectionsamongpregnantNigerianwomen. Instituto Oswaldo Cruz. Rio de Janeiro, v. 96, n. 8, p. 1055-1059, nov. 2001.

FAILACE, R. Hemograma: Manual de Interpretação. 3. ed., Porto Alegre: Artes Médicas, 1995.

FAUST, E. C. et al. Comparative efficiency of various techniques for the diagnosis of protozoa and helminth in feces.The Journal of Parasitology, New Orleans, v. 25, n. 3, p. 241-262, jun. 1939.

FERRO FERENE. Farmacêutico responsável: Jorge A. Janoni. Bula de reagentes para determinação quantitativa de ferro em soro.São Gonçalo, RJ: Kovalent do Brasil LTDA, 2008 .

FINKEISTEIN, J. L. et al. Predictors of anaemia and iron deficiency in HIV-infected pregnant women in Tanzania: a potencial role for vitamin D and parasitic infections.Public Health Nutrition, v. 15, n. 5, p. 928-937, maio 2012.

GLICOSE GOD-PAP. Farmacêutico responsável: Jorge A. Janoni. Bula de reagentes para determinação quantitativa de glicose em soro ou plasma.São Gonçalo, RJ: Kovalent do Brasil LTDA, 2010.

HEDRICH, A. et al. Perfil alimentar, estado nutricional, de saúde e condições sócio-econômicas de gestantes assistidas por centros de saúde do município de Guarapuava-PR. Revista Salus, Guarapuava, v. 1, n. 2, p. 139-146, jul.-dez. 2007.

HOFFMAN, W. A.; PONS, J. A.; JANER, J. L.The sedimentation method in schistosomiasis mansoni.Puerto 
Rico Journal of Public Health and Tropical Medicine, v. 9, p. 281-98, 1934.

IOM \& NRC. Institute of Medicine and National Research Council. Weight Gain During Pregnancy: Reexamining the Guidelines. The National Academies Press, Washington, DC, 2009.

LANGER, O. Fetal macrosomia: etiologic factors. Clinical Obstetrics and Gynecology.New York, v. 43, n. 2, p. 283297, 2000.

LIMA, G. S. P.; SAMPAIO, H. A. C. Influencia de fatores obstétricos, socioeconômicos e nutricionais da gestante sobre o peso do recém-nascido: estudo realizado em uma maternidade em Teresina, Piauí. Revista Brasileira deSaúde Materno Infantil,Recife, v. 4, n. 3, p. 253-261, jul.-set. 2004.

MACEDO, L. M. C.; REY, L. Enteroparasitoses em gestantes e puérperas no Rio de Janeiro. Caderno de Saúde Pública, Rio de Janeiro, v. 12, n. 3, p. 183-388, jul-set. 1996.

MARTINEZ, I., BELDA NETO, F. M. Contribution to the laboratory diagnosis of human cryptosporidiosis. Revista do Instituto de Medicina Tropical de São Paulo. São Paulo, v. 43, n. 2, p. 79-82, mar./abr. 2001.

MINÉ, J. C. Caracterização de cepas de Strongyloidesstercoralis (Bavay, 1876) Stiles \&Hassall, 1902 (Rhabditida, Strongyloididae) isoladas de pacientes com e sem sintomatologia da região de Araraquara-SP. Araraquara, 2009. 129 p. Tese (Doutorado em Biociências e Biotecnologia Aplicadas à Farmácia - Área de Parasitologia) - Universidade Estadual Paulista, Araraquara, 2009.

NEVES, D. P. Parasitologia Humana. 11. ed., São Paulo: Atheneu, 2005.

NOGUEIRA, A.I.et al. Diabetes Gestacional: perfil e evolução de um grupo de pacientes do Hospital das Clínicas da UFMG. Revista Médica de Minas Gerais, Belo Horizonte, v. 21, n. 1, p. 32-41, 2011.

PADILHA, P. C. et al. Associação entre o estado nutricional pré-gestacional e a predição do risco de intercorrências gestacionais. Revista Brasileira de Ginecologia e Obstetrícia, Rio de Janeiro, v. 29, n. 10, p. 511-518. 2007.

PONTES, M. H.Avaliação do estado nutricional das gestantes assistidas no serviço pré-natal em uma unidade primária de saúde de Fortaleza. Monografia Curso de Especialização em Nutrição Materno-Infantil e do Adolescente, Fortaleza, 2007.

REZENDE, F. A. C. et al.Aplicabilidade do Índice de Massa Corporal na avaliação da gordura corporal. Revista Brasileira Medicina do Esporte, v. 16, n. 2, mar/abr. 2010.

RUDGE, M. V. C; CALDERON, I. M. P. Macrossomia fetal: correlação clínica-experimental. Femina, v. 25, n. 5, p. 469-76., 1997.
ROCHA, G. K. A. M. et al. Prevalência de anemias em crianças e adolescentes portadores de enteroparasitoses. NewsLab, São Paulo, v. 65, p.172-188, 2004.

ROCHA, D. S. et al. Estado nutricional e anemia ferropriva em gestantes: relação com o peso da criança ao nascer. Revista de Nutrição,Campinas, v. 18, n. 4, p. 481-489, jul./ ago. 2005.

ROSSO, P. R; MARDONDES, F. S. Gráfica de incremento de peso para embarazadas. Ministério de Salud, Chile, 1986.

RUGAI, E.; MATTOS, T.; BRISOLA, A. Nova técnica para isolar larvas de nematóides das fezes - modificação do método de Baermann. Revista do Instituto Adolfo Lutz, v. 14, p. 5-8, 1954.

SBEM. Sociedade Brasileira de Endocrinologia e Metabologia.Sobrepeso e Obesidade: Diagnóstico.Projeto Diretrizes. Associação Médica Brasileira e Conselho Federal de Medicina, p. 4,ago. 2004.

SOUZA, A. I. et al. Enteroparasitoses, anemia e estado nutricional em grávidas atendidas em serviço público de saúde. Revista Brasileira de Ginecologia e Obstetrícia. Rio de Janeiro, v. 24, n. 4, maio 2002.

SOUZA, A. I.;BATISTA FILHO, M.; FERREIRA, L. O. C. Alterações hematológicas e gravidez. Revista Brasileira de Hematologia e Hemoterapia, Recife, v. 24, n. 1, p. 29-36, 2002.

YATICH, N. J. et al. Malaria and intestinal helminth coinfection among pregnant women in Ghana: prevalence and risk factors. American Society of Tropical Medicine and Hygiene, v. 80, n. 6, p. 896-901, 2009.

* Créditos de revisão de língua portuguesa: Maria Antonia B. M. Wakabara. 